arXiv: 0709.2277 [hep-th]

CAS-BHU/Preprint

\title{
NILPOTENT SYMMETRY INVARIANCE IN THE NON-ABELIAN 1-FORM GAUGE THEORY: SUPERFIELD FORMALISM
}

\author{
R. P. Malik 11 and B. P. Mandal \\ Physics Department, Centre of Advanced Studies, \\ Banaras Hindu University, Varanasi-221 005, (U. P.), India \\ E-mails: malik@bhu.ac.in; bhabani@bhu.ac.in
}

\begin{abstract}
We demonstrate that the nilpotent Becchi-Rouet-Stora-Tyutin (BRST) and anti-BRST symmetry invariance of the Lagrangian density of a four $(3+1)$-dimensional (4D) non-Abelian 1-form gauge theory with Dirac fields can be captured within the framework of the superfield approach to BRST formalism. The above 4D theory, where there is an explicit coupling between the non-Abelian 1-form gauge field and the Dirac fields, is considered on a (4, 2)-dimensional supermanifold, parameterized by the bosonic 4D space-time variables and a pair of Grassmannian variables. We show that the Grassmannian independence of the super Lagrangian density, expressed in terms of the $(4,2)$-dimensional superfields, is a clear signature of the presence of the (anti-)BRST invariance in the original 4D theory.
\end{abstract}

PACS numbers: 11.15.-q, 03.70.+k

Keywords: Non-Abelian 1-form gauge theory, Dirac fields, (Anti-)BRST invariance, Superfield formalism, Geometrical interpretations

\footnotetext{
${ }^{1}$ Presently also associated with the DST-Centre for Interdisciplinary Mathematical Sciences, Faculty of Science, Banaras Hindu University, Varanasi - 221 005, (U. P.), India.
} 


\section{Introduction}

The geometrical interpretations of the nilpotency and anticommutativity properties, associated with the (anti-)BRST symmetry transformations of the gauge and (anti-)ghost fields of a given 1-form gauge theory, have been provided within the framework of the usual superfield approach to BRST formalism [1-9]. This approach, however, has not been able to shed any light on the nilpotent (anti-)BRST symmetry transformations of the matter fields of an interacting (non-)Abelian 1-form gauge theory.

In a set of research papers [10-20], the above superfield formulation [1-9], has been consistently extended so as to derive the nilpotent (anti-)BRST symmetry transformations for the matter (i.e. Dirac, complex scalar, etc.) fields of a given (non-)Abelian 1-form gauge theory. In the above attempts [1-20], however, the (anti-)BRST invariance of the Lagrangian densities has not yet been captured within the framework of the superfield formulation.

The central theme of a couple of very recent papers [21,22] concerns itself with the (anti-)BRST invariance of the 4D (non-)Abelian 1-form gauge theories within the framework of the superfield approach to BRST formalism. To be precise, it has been shown that the Grassmannian independence of the super Lagrangian density, expressed in terms of the superfields defined on the $(4,2)$-dimensional supermanifold, is a clear-cut signature of the presence of the (anti-)BRST invariance in the above 1-form gauge theories.

The field theoretical models that have been considered in $[21,22]$ are (i) the 4D (non-)Abelian gauge theory without any interaction with matter fields [21], and (ii) the interacting Abelian U(1) 1-form gauge theory with Dirac fields [22]. The purpose of our present investigation is to generalize our results of $[21,22]$ to the case of the interacting $4 \mathrm{D}$ non-Abelian gauge theory (with Dirac fields). Furthermore, we demonstrate that, like our earlier observations in $[21,22]$, the presence of the (anti-)BRST invariance in the 4D ordinary nonAbelian 1-form gauge theory is encoded in the proof that the Grassmannian derivatives, acting on the super Lagrangian density, produce zero result.

The motivating factors that have propelled us to pursue our present investigation are as follows. First and foremost, it is important to check the validity of our results (that were obtained for the (non-)interacting Abelian 1-form gauge theory) to our present model of the interacting non-Abelian theory. Second, it is interesting to explore the geometrical interpretation of the (anti-)BRST invariance for our present interacting non-Abelian gauge theory which were found to be quite cute and simple for the interacting $\mathrm{U}(1)$ gauge theory (with Dirac fields). Finally, our earlier works [21,22] and our present endeavour are our modest steps towards our main goal of applying the superfield formulation to the 2-form (and still higher form) gauge theories. 
The material of our present investigation is organized as follows. In Sec. 2 , we recapitulate the bare essentials of the (anti-)BRST invariance of our present interacting non-Abelian theory in the Lagrangian formulation. Section 3 is devoted to a brief discussion of the horizontality condition and its consequences within the framework of the superfield formulation. Our Sec. 4 deals with a gauge invariant restriction (GIR) on the matter superfields of the (4, 2)-dimensional supermanifold and its outcome for the (anti-)BRST invariance in our present 4D theory. In Sec. 5, we provide a concise discussion of a single GIR on the matter superfields that leads to the consequences of our Secs. 3 and 4. Finally, we make some concluding remarks in Sec. 6 .

\section{Off-shell nilpotent (anti-)BRST symmetry invariance: Lagrangian formulation}

Let us begin with the following Lagrangian densities for the 4D interacting non-Abelian 1-form gauge theory with Dirac fields in the Feynman gauge [23]

$$
\begin{aligned}
\mathcal{L}_{b}^{(1)} & =-\frac{1}{4} F^{\mu \nu} \cdot F_{\mu \nu}+\bar{\psi}\left(i \gamma^{\mu} D_{\mu}-m\right) \psi+B \cdot\left(\partial_{\mu} A^{\mu}\right) \\
& +\frac{1}{2}(B \cdot B+\bar{B} \cdot \bar{B})-i \partial_{\mu} \bar{C} \cdot D^{\mu} C, \\
\mathcal{L}_{\bar{b}}^{(2)} & =-\frac{1}{4} F^{\mu \nu} \cdot F_{\mu \nu}+\bar{\psi}\left(i \gamma^{\mu} D_{\mu}-m\right) \psi-\bar{B} \cdot\left(\partial_{\mu} A^{\mu}\right) \\
& +\frac{1}{2}(B \cdot B+\bar{B} \cdot \bar{B})-i D_{\mu} \bar{C} \cdot \partial^{\mu} C,
\end{aligned}
$$

where $D_{\mu} C=\partial_{\mu} C+i A_{\mu} \times C$ and $D_{\mu} \psi=\partial_{\mu} \psi+i\left(A_{\mu} \cdot T\right) \psi$ are the covariant derivatives on the fermionic ghost field $C$ and matter (Dirac) field $\psi$, respectively. These covariant derivatives satisfy $\left[D_{\mu}, D_{\nu}\right] \psi=i F_{\mu \nu} \psi,\left[D_{\mu}, D_{\nu}\right] C=$ $i F_{\mu \nu} \times C$ which define 2 the curvature tensor $F_{\mu \nu}=\partial_{\mu} A_{\nu}-\partial_{\nu} A_{\mu}+i A_{\mu} \times A_{\nu}$. Here $B$ and $\bar{B}$ are the auxiliary fields that satisfy the Curci-Ferrari condition $B+\bar{B}=-(C \times \bar{C})[24]$ so as to make the following off-shell nilpotent $\left(s_{(a) b}^{2}=0\right)\left(\right.$ anti-)BRST symmetry transformations $s_{(a) b}$ (see, e.g [23])

$$
s_{b} A_{\mu}=D_{\mu} C, \quad s_{b} C=-\frac{i}{2}(C \times C), \quad s_{b} \bar{C}=i B, \quad s_{b} B=0,
$$

\footnotetext{
${ }^{2}$ We adopt here the conventions and notations such that the $4 \mathrm{D}$ flat metric $\eta_{\mu \nu}$ has the signature $(+1,-1,-1,-1)$ and dot and cross products between two vectors $P^{a}$ and $Q^{a}$ in the Lie algebraic group space are $P \cdot Q=P^{a} Q^{a}$ and $(P \times Q)^{a}=f^{a b c} P^{b} Q^{c}$ where $f^{a b c}$ are the structure constants in the $S U(N)$ Lie algebra $\left[T^{a}, T^{b}\right]=f^{a b c} T^{c}$ obeyed by the generators $T^{a}$ 's. The latter are present in the definition of the non-Abelian 1-form $A^{(1)}=d x^{\mu} A_{\mu} \cdot T$. In the above, we have: $\mu, \nu \ldots=0,1,2,3$ and $a, b, c=1,2,3 \ldots N$.
} 


$$
\begin{gathered}
s_{b} \psi=-i(C \cdot T) \psi, \quad s_{b} \bar{\psi}=-i \bar{\psi}(C \cdot T), \quad s_{b} \bar{B}=i(\bar{B} \times C), \\
s_{a b} A_{\mu}=D_{\mu} \bar{C}, \quad s_{a b} \bar{C}=-\frac{i}{2}(\bar{C} \times \bar{C}), \quad s_{a b} C=i \bar{B}, \quad s_{a b} \bar{B}=0, \\
s_{a b} \psi=-i(\bar{C} \cdot T) \psi, \quad s_{a b} \bar{\psi}=-i \bar{\psi}(\bar{C} \cdot T), \quad s_{a b} B=i(B \times \bar{C}),
\end{gathered}
$$

anticommutative $\left(s_{b} s_{a b}+s_{a b} s_{b}=0\right)$ in nature. In the above, the fields $\bar{C}^{a}\left(C^{a}\right)$ are the anticommuting (anti-)ghost fields that are required for the proof of unitarity in the theory [25] and $\gamma^{\mu}$ are the usual $4 \times 4$ Dirac matrices.

The above nilpotent transformations (3) and (4) are the symmetry transformations because the Lagrangian densities change to total derivatives under them. The key reasons behind the (anti-)BRST invariance are (i) the symmetry invariance of the kinetic energy term (i.e. $s_{(a) b}\left[F^{\mu \nu} \cdot F_{\mu \nu}\right]=0$ ), (ii) the invariance of the terms that contain Dirac fields (i.e. $s_{(a) b}\left[\bar{\psi}\left(i \gamma^{\mu} D_{\mu}-m\right) \psi\right]=0$ ), and (iii) the invariance of the gauge-fixing and Faddeev-Popov ghost term. In fact, the last statement can be mathematically expressed as

$$
\begin{aligned}
& s_{b} s_{a b}\left[\frac{i}{2} A_{\mu} \cdot A^{\mu}+C \cdot \bar{C}\right]=B \cdot\left(\partial_{\mu} A^{\mu}\right)+\frac{1}{2}(B \cdot B+\bar{B} \cdot \bar{B})-i \partial_{\mu} \bar{C} \cdot D^{\mu} C \\
& \equiv-\bar{B} \cdot\left(\partial_{\mu} A^{\mu}\right)+\frac{1}{2}(B \cdot B+\bar{B} \cdot \bar{B})-i D_{\mu} \bar{C} \cdot \partial^{\mu} C
\end{aligned}
$$

The above expression clearly implies (due to the nilpotency and anticommutativity of $\left.s_{(a) b}\right)$ that the gauge-fixing and Faddeev-Popov ghost terms together remain invariant under the (anti-)BRST symmetry transformations.

\section{Horizontality condition: outcomes}

To tap the potential and power of the celebrated horizontality condition (HC), first of all, we generalize the ordinary exterior derivative $d=d x^{\mu} \partial_{\mu}$ and the ordinary 1-form connection $\left(A^{(1)}=d x^{\mu} A_{\mu}\right)$ of the $4 \mathrm{D}$ theory to their counterparts on the $(4,2)$-dimensional supermanifold as follows

$$
\begin{array}{r}
d \rightarrow \tilde{d}=d x^{\mu} \partial_{\mu}+d \theta \partial_{\theta}+d \bar{\theta} \partial_{\bar{\theta}}, \\
A^{(1)} \rightarrow \tilde{A}^{(1)}=d x^{\mu}\left(\mathcal{B}_{\mu} \cdot T\right)+d \theta(\overline{\mathcal{F}} \cdot T)+d \bar{\theta}(\mathcal{F} \cdot T),
\end{array}
$$

where $\left(\mathcal{B}_{\mu}, \mathcal{F}, \overline{\mathcal{F}}\right)$ are the superfields defined on the above supermanifold. These are the generalization of the basic fields $\left(A_{\mu}, C, \bar{C}\right)$ as can be seen from the following expansion along the Grassmannian directions $[4,5,16]$

$$
\begin{aligned}
\mathcal{B}_{\mu}(x, \theta, \bar{\theta}) & =\left(A_{\mu} \cdot T\right)(x)+\theta\left(\bar{R}_{\mu} \cdot T\right)(x)+\bar{\theta}\left(R_{\mu} \cdot T\right)(x)+i \theta \bar{\theta}\left(S_{\mu} \cdot T\right)(x), \\
\mathcal{F}(x, \theta, \bar{\theta}) & =(C \cdot T)(x)+i \theta(\bar{B} \cdot T)(x)+i \bar{\theta}(\mathcal{B} \cdot T)(x)+i \theta \bar{\theta}(s \cdot T)(x), \\
\overline{\mathcal{F}}(x, \theta, \bar{\theta}) & =(\bar{C} \cdot T)(x)+i \theta(\overline{\mathcal{B}} \cdot T)(x)+i \bar{\theta}(B \cdot T)(x)+i \theta \bar{\theta}(\bar{s} \cdot T)(x) .
\end{aligned}
$$


It is elementary to check that, in the limit $(\theta, \bar{\theta}) \rightarrow 0$, we retrieve basic $4 \mathrm{D}$ local fields $A_{\mu}, C$ and $\bar{C}$ of our Lagrangian densities (1) and/or (2).

The $\mathrm{HC}$ is the requirement that the super 2 -form $\tilde{F}^{(2)}=\tilde{d} \tilde{A}^{(1)}+i \tilde{A}^{(1)} \wedge \tilde{A}^{(1)}$ is equal to the ordinary 2-form $F^{(2)}=d A^{(1)}+i A^{(1)} \wedge A^{(1)}$. This equality leads to the determination of the secondary fields $R_{\mu}, \bar{R}_{\mu}, S_{\mu}, \mathcal{B}, \overline{\mathcal{B}}, s, \bar{s}$ of the expansion (7) in terms of the basic fields (see, e.g. [16]). The ensuing expansion, with these values suitably inserted into (7), looks as 3

$$
\begin{aligned}
\mathcal{B}_{\mu}^{(h)}(x, \theta, \bar{\theta}) & =A_{\mu}+\theta D_{\mu} \bar{C}+\bar{\theta} D_{\mu} C+i \theta \bar{\theta}\left(D_{\mu} B+D_{\mu} C \times \bar{C}\right) \\
& \equiv A_{\mu}(x)+\theta\left(s_{a b} A_{\mu}(x)\right)+\bar{\theta}\left(s_{b} A_{\mu}(x)\right)+\theta \bar{\theta}\left(s_{b} s_{a b} A_{\mu}(x)\right), \\
\mathcal{F}^{(h)}(x, \theta, \bar{\theta}) & =C+i \theta \bar{B}-\frac{i}{2} \bar{\theta}(C \times C)-\theta \bar{\theta}(\bar{B} \times C) \\
& \equiv C(x)+\theta\left(s_{a b} C(x)\right)+\bar{\theta}\left(s_{b} C(x)\right)+\theta \bar{\theta}\left(s_{b} s_{a b} C(x)\right), \\
\overline{\mathcal{F}}^{(h)}(x, \theta, \bar{\theta}) & =\bar{C}-\frac{i}{2} \theta(\bar{C} \times \bar{C})+i \bar{\theta} B+\theta \bar{\theta}(B \times \bar{C}) \\
& \equiv \bar{C}(x)+\theta\left(s_{a b} \bar{C}(x)\right)+\bar{\theta}\left(s_{b} \bar{C}(x)\right)+\theta \bar{\theta}\left(s_{b} s_{a b} \bar{C}(x)\right) .
\end{aligned}
$$

In the above, the superscript $(h)$ stands for the superfields that are obtained after the application of the $\mathrm{HC}$ and $s_{(a) b}$ are the transformations (3) and (4).

It is evident that the (anti-)BRST symmetry transformations can be expressed as: $s_{b} \Omega=\operatorname{Lim}_{\theta \rightarrow 0}(\partial / \partial \bar{\theta}) \tilde{\Omega}^{(h)}, s_{a b} \Omega=\operatorname{Lim}_{\bar{\theta} \rightarrow 0}(\partial / \partial \theta) \tilde{\Omega}^{(h)}$. Here the local $4 \mathrm{D}$ generic field is $\Omega(x)$ and its counterpart on the $(4,2)$-dimensional supermanifold is the superfield $\tilde{\Omega}^{(h)}$ (obtained after the application of HC). The above mapping provides the geometrical interpretation of the nilpotent (anti)BRST transformations $s_{(a) b}$ as the translational generators $\left(\partial_{\theta}, \partial_{\bar{\theta}}\right)$ along the Grassmannian directions of the (4, 2)-dimensional supermanifold.

The 2-form super curvature tensor $\tilde{F}_{\mu \nu}^{(h)}=\partial_{\mu} \mathcal{B}_{\nu}^{(h)}-\partial_{\nu} \mathcal{B}_{\mu}^{(h)}+i \mathcal{B}_{\mu}^{(h)} \wedge \mathcal{B}_{\nu}^{(h)}$ can be explicitly expanded along the Grassmannian directions as [4]

$$
\tilde{F}_{\mu \nu}^{(h)}=F_{\mu \nu}+i \theta\left(F_{\mu \nu} \times \bar{C}\right)+i \bar{\theta}\left(F_{\mu \nu} \times C\right)-\theta \bar{\theta}\left(F_{\mu \nu} \times B+F_{\mu \nu} \times C \times \bar{C}\right) .
$$

The above equation immediately implies that the kinetic energy term of the Lagrangian densities (1) and/or (2) remains unaffected due to presence of the Grassmannian variables, namely; (see, e.g. [4] for details)

$$
-\frac{1}{4} \tilde{F}^{\mu \nu(h)} \cdot \tilde{F}_{\mu \nu}^{(h)}=-\frac{1}{4} F^{\mu \nu} \cdot F_{\mu \nu} .
$$

In the above proof, it is the structure constants $f^{a b c}$ (which are chosen to be totally antisymmetric [23] for the $\mathrm{SU}(\mathrm{N})$ group) play an important role.

\footnotetext{
${ }^{3}$ For the sake of brevity, we use the notations $A_{\mu} \equiv A_{\mu} \cdot T, C=C \cdot T$, etc.
} 
Physically, the above equality shows that the l.h.s. is, ultimately, indepen$\operatorname{dent}\left(\partial_{\theta}\left[\tilde{F}^{\mu \nu(h)} \cdot \tilde{F}_{\mu \nu}^{(h)}\right]=0, \partial_{\bar{\theta}}\left[\tilde{F}^{\mu \nu(h)} \cdot \tilde{F}_{\mu \nu}^{(h)}\right]=0\right)$ of the Grassmannian variables $\theta$ and $\bar{\theta}$. This observation, in turn, implies the (anti-)BRST invariance of the 4D kinetic energy term in the framework of superfield approach to BRST formalism because of the mappings: $s_{b} \Leftrightarrow \operatorname{Lim}_{\theta \rightarrow 0} \partial_{\bar{\theta}}, s_{a b} \Leftrightarrow \operatorname{Lim}_{\bar{\theta} \rightarrow 0} \partial_{\theta}$.

In an exactly similar fashion, it can be checked that the gauge-fixing and Faddeev-Popov ghost terms of the theory (cf. (5)) can be expressed in terms of the Grassmannian derivatives $\left(\partial_{\theta}, \partial_{\bar{\theta}}\right)$ and the superfields (obtained after the application of $\mathrm{HC}$ ) as

$$
s_{b} s_{a b}\left[\frac{i}{2} A_{\mu} \cdot A^{\mu}+C \cdot \bar{C}\right]=\frac{\partial}{\partial \bar{\theta}} \frac{\partial}{\partial \theta}\left[\frac{i}{2} \mathcal{B}_{\mu}^{(h)} \cdot \mathcal{B}^{\mu(h)}+\mathcal{F}^{(h)} \cdot \overline{\mathcal{F}}^{(h)}\right],
$$

where the superfields, with superscript $(h)$, are listed in (8). It is now elementary to the check that the following super Lagrangian density $\left(\tilde{\mathcal{L}}_{M}\right)$, containing kinetic energy, gauge-fixing and Faddeev-Popov ghost terms, namely;

$$
\tilde{\mathcal{L}}_{M}=-\frac{1}{4} \tilde{F}^{\mu \nu(h)} \cdot \tilde{F}_{\mu \nu}^{(h)}+\frac{\partial}{\partial \bar{\theta}} \frac{\partial}{\partial \theta}\left[\frac{i}{2} \mathcal{B}_{\mu}^{(h)} \cdot \mathcal{B}^{\mu(h)}+\mathcal{F}^{(h)} \cdot \overline{\mathcal{F}}^{(h)}\right],
$$

is the counterpart of its $4 \mathrm{D}$ analogue that is represented by the following Lagrangian density (i.e. $\mathcal{L}_{M}$ )

$$
\begin{aligned}
& \mathcal{L}_{M}=-\frac{1}{4} F^{\mu \nu} \cdot F_{\mu \nu}-\bar{B} \cdot\left(\partial_{\mu} A^{\mu}\right)+\frac{1}{2}(B \cdot B+\bar{B} \cdot \bar{B})-i D_{\mu} \bar{C} \cdot \partial^{\mu} C \\
& \equiv-\frac{1}{4} F^{\mu \nu} \cdot F_{\mu \nu}+B \cdot\left(\partial_{\mu} A^{\mu}\right)+\frac{1}{2}(B \cdot B+\bar{B} \cdot \bar{B})-i \partial_{\mu} \bar{C} \cdot D^{\mu} C .
\end{aligned}
$$

The above Lagrangian density is a part of the Lagrangian densities (1) and (2). One of the decisive consequences of the HC is that the super Lagrangian density $\tilde{\mathcal{L}}_{M}$ is independent of the Grassmannian variables because $\operatorname{Lim}_{\theta \rightarrow 0} \partial_{\bar{\theta}} \tilde{\mathcal{L}}_{M}=0$ and $\operatorname{Lim}_{\bar{\theta} \rightarrow 0} \partial_{\theta} \tilde{\mathcal{L}}_{M}=0$. This key statement is equivalent to the (anti-)BRST invariance of the kinetic energy, gauge-fixing and FaddeevPopov ghost terms of the 4D Lagrangian density of our present theory.

Mathematically, the above correspondence can be succinctly expressed as

$$
\operatorname{Lim}_{\theta \rightarrow 0} \frac{\partial}{\partial \bar{\theta}} \tilde{\mathcal{L}}_{M}=0 \Leftrightarrow s_{b} \mathcal{L}_{M}=0, \quad \operatorname{Lim}_{\bar{\theta} \rightarrow 0} \frac{\partial}{\partial \theta} \tilde{\mathcal{L}}_{M}=0 \Leftrightarrow s_{a b} \mathcal{L}_{M}=0 .
$$

This mapping captures, in a very simple manner, the (anti-)BRST invariance of the kinetic energy, gauge-fixing and Faddeev-Popov ghost terms of the Lagrangian density within the framework of the superfield formalism. In other words, if the action of the Grassmannian derivatives on the super Lagrangian density happens to be zero, the corresponding 4D Lagrangian density would respect the (anti-)BRST invariance. In the above proof (cf. (14)), the nilpotency (i.e. $\partial_{\theta}^{2}=0, \partial_{\bar{\theta}}^{2}=0$ ) and anticommutativity (i.e. $\partial_{\theta} \partial_{\bar{\theta}}+\partial_{\bar{\theta}} \partial_{\theta}=0$ ) of the translational generators along Grassmannian directions play key roles. 


\section{Gauge invariant restriction: consequences}

To obtain the (anti-)BRST symmetry transformations for the matter fields of the theory, we exploit the following gauge invariant restriction (GIR) on the matter superfields [16]

$$
\bar{\Psi}(x, \theta, \bar{\theta})\left[\tilde{d}+i \tilde{A}^{(1)(h)}\right] \Psi(x, \theta, \bar{\theta})=\bar{\psi}(x)\left[d+i A^{(1)}\right] \psi(x),
$$

where $\tilde{A}^{(1)(h)}=d x^{\mu} \mathcal{B}_{\mu}^{(h)}+d \theta \overline{\mathcal{F}}^{(h)}+d \bar{\theta} \mathcal{F}^{(h)}$ is the super 1-form connection expressed in terms of the superfields listed in (8). It is interesting to note that, in the above unique relationship, the $\mathrm{HC}$ and matter (super) fields are intertwined in a gauge (i.e. BRST) invariant manner.

The matter superfields $(\Psi, \bar{\Psi})$ are the generalizations of the 4D Dirac fields $(\psi, \bar{\psi})$ of the Lagrangian densities (1) and/or (2) as can be seen from the following expansion

$$
\begin{aligned}
& \Psi(x, \theta, \bar{\theta})=\psi(x)+i \theta\left(b_{1} \cdot T\right)+i \bar{\theta}\left(\bar{b}_{1} \cdot T\right)+i \theta \bar{\theta}(f \cdot T), \\
& \bar{\Psi}(x, \theta, \bar{\theta})=\bar{\psi}(x)+i \theta\left(b_{2} \cdot T\right)+i \bar{\theta}\left(\bar{b}_{2} \cdot T\right)+i \theta \bar{\theta}(\bar{f} \cdot T),
\end{aligned}
$$

where the secondary fields $b_{1}, \bar{b}_{1}, b_{2}, \bar{b}_{2}, f, \bar{f}$ are determined in terms of the basic fields from the GIR (15). These expressions are as follows $[16,19]$

$$
\begin{aligned}
& b_{1}=-(C \cdot T) \psi, \bar{b}_{1}=-(\bar{C} \cdot T) \psi, b_{2}=-\bar{\psi}(C \cdot T), \bar{b}_{2}=-\bar{\psi}(\bar{C} \cdot T), \\
& f=-i\left[B+\frac{1}{2}(C \times \bar{C})\right] \psi, \quad \bar{f}=+i \bar{\psi}\left[B+\frac{1}{2}(C \times \bar{C})\right] .
\end{aligned}
$$

Insertions of the above values into the expansion (16) leads to [16-19]

$$
\begin{aligned}
\Psi^{(G)}(x, \theta, \bar{\theta}) & =\psi(x)+\theta(-i \bar{C} \cdot T \psi)+\bar{\theta}(-i C \cdot T \psi)+\theta \bar{\theta}\left(B+\frac{1}{2} C \times \bar{C}\right) \psi \\
& \equiv \psi(x)+\theta\left(s_{a b} \psi(x)\right)+\bar{\theta}\left(s_{b} \psi(x)\right)+\theta \bar{\theta}\left(s_{b} s_{a b} \psi(x)\right) \\
\bar{\Psi}^{(G)}(x, \theta, \bar{\theta}) & =\bar{\psi}+\theta(-i \bar{\psi} \bar{C} \cdot T)+\bar{\theta}(-i \bar{\psi} C \cdot T)-\theta \bar{\theta} \bar{\psi}\left(B+\frac{1}{2} C \times \bar{C}\right) \\
& \equiv \bar{\psi}(x)+\theta\left(s_{a b} \bar{\psi}(x)\right)+\bar{\theta}\left(s_{b} \bar{\psi}(x)\right)+\theta \bar{\theta}\left(s_{b} s_{a b} \bar{\psi}(x)\right)
\end{aligned}
$$

where the nilpotent transformations $s_{(a) b}$ are listed in (3) and (4) and the superscript $(G)$ on the above matter superfields denotes that these superfields have been obtained after the application of GIR.

As a consequence of the above expansion (18) (that has been obtained after the application of the GIR in (15)), it is clear that the following equality (that would be useful for our discussions) is true, namely;

$$
\bar{\Psi}^{(G)}(x, \theta, \bar{\theta}) \Psi^{(G)}(x, \theta, \bar{\theta})=\bar{\psi}(x) \psi(x) .
$$


Furthermore, it can be checked that the following key equality is also valid on the matter superfields (after the application of GIR and HC):

$$
\bar{\Psi}^{(G)}\left[i \gamma^{M} D_{M}^{(h)}-m\right] \Psi^{(G)}=\bar{\psi}(x)\left(i \gamma^{\mu} D_{\mu}-m\right) \psi(x) \equiv \mathcal{L}_{d},
$$

where $\mathcal{L}_{d}$ is the $4 \mathrm{D}$ Lagrangian density that contains Dirac fields and $\gamma^{M}$ are the generalizations of the $4 \times 4$ Dirac matrices onto $(4,2)$-dimensional supermanifold. With the specific choice of $\gamma^{M}=\left(\gamma^{\mu}, C_{\theta}, C_{\bar{\theta}}\right)$, we obtain

$$
\gamma^{M} D_{M}^{(h)}=\gamma^{\mu}\left(\partial_{\mu}+i \mathcal{B}_{\mu}^{(h)}\right)+C_{\theta}\left(\partial_{\theta}+i \overline{\mathcal{F}}^{(h)}\right)+C_{\bar{\theta}}\left(\partial_{\bar{\theta}}+i \mathcal{F}^{(h)}\right),
$$

where $C_{\theta}$ and $C_{\bar{\theta}}$ are some anticommuting $\left(C_{\theta}^{2}=0, C_{\bar{\theta}}^{2}=0, C_{\theta} C_{\bar{\theta}}+C_{\bar{\theta}} C_{\theta}=0\right)$ constants which go to zero (i.e. $C_{\theta} \rightarrow 0, C_{\bar{\theta}} \rightarrow 0$ ) in the limiting case of $(\theta, \bar{\theta}) \rightarrow 0$. These requirements on $C_{\theta}$ and $C_{\bar{\theta}}$ are essential so as to maintain the bosonic nature of the r.h.s. and to prove that:

$$
\operatorname{Lim}_{(\theta, \bar{\theta}) \rightarrow 0} \gamma^{M} D_{M}=\gamma^{\mu} D_{\mu} \equiv \gamma^{\mu}\left(\partial_{\mu}+i A_{\mu} \cdot T\right) .
$$

The above equation implies that, ultimately, we obtain the ordinary 4D Dirac Lagrangian density when the Grassmannian variables are set equal to zero.

The exact mathematical form of the constants $C_{\theta}$ and $C_{\bar{\theta}}$ is not important for our present discussions because, irrespective of their form, the following equations (with the superfields $\mathcal{F}^{(h)}, \overline{\mathcal{F}}^{(h)}, \Psi^{(G)}$ ) are always satisfied

$$
\left(\partial_{\theta}+i \overline{\mathcal{F}}^{(h)}\right) \Psi^{(G)}=0, \quad\left(\partial_{\bar{\theta}}+i \mathcal{F}^{(h)}\right) \Psi^{(G)}=0 .
$$

As a consequence, the exact mathematical form of the anticommuting constants $C_{\theta}$ and $C_{\bar{\theta}}$ does not affect the key results that emerge from the equation (20) which happens to be a GIR on the matter superfields of the theory. With inputs from (23), it is clear that the condition (20) reduces to

$$
\bar{\Psi}^{(G)}\left[i \gamma^{\mu}\left(\partial_{\mu}+i \mathcal{B}_{\mu}^{(h)}\right)-m\right] \Psi^{(G)}=\bar{\psi}(x)\left(i \gamma^{\mu} D_{\mu}-m\right) \psi(x) .
$$

The above equation is readily satisfied if we insert the superfield expansions (8) and (18) that have been obtained after the application of HC and GIR.

It is clear from the equation $(24)$ that the super Lagrangian density $\left(\tilde{\mathcal{L}}_{d}\right)$ with gauge and matter superfields and the ordinary Lagrangian density $\left(\mathcal{L}_{d}\right)$ with gauge and Dirac ordinary fields, namely;

$$
\tilde{\mathcal{L}}_{d}=\bar{\Psi}^{(G)}\left[i \gamma^{\mu}\left(\partial_{\mu}+i \mathcal{B}_{\mu}^{(h)}\right)-m\right] \Psi^{(G)}, \quad \mathcal{L}_{d}=\bar{\psi}(x)\left(i \gamma^{\mu} D_{\mu}-m\right) \psi(x),
$$

are equal in the sense that the $(4,2)$-dimensional super Lagrangian density $\tilde{\mathcal{L}}_{d}$ is effectively independent of the Grassmannian variables $\theta$ and $\bar{\theta}$. Thus, the (anti-)BRST invariance can be expressed by the following mappings

$$
\operatorname{Lim}_{\theta \rightarrow 0} \frac{\partial}{\partial \bar{\theta}} \tilde{\mathcal{L}}_{d}=0 \Leftrightarrow s_{b} \mathcal{L}_{d}=0, \quad \operatorname{Lim}_{\bar{\theta} \rightarrow 0} \frac{\partial}{\partial \theta} \tilde{\mathcal{L}}_{d}=0 \Leftrightarrow s_{a b} \mathcal{L}_{d}=0 .
$$


Here $s_{(a) b}$ are the transformations that are given in (3) and (4).

It is worthwhile to recall that the ordinary Lagrangian density $\mathcal{L}_{d}$ remains invariant (i.e. $s_{(a) b}\left[\bar{\psi}\left(i \gamma^{\mu} \partial_{\mu}-m\right) \psi=0\right.$ ) under the symmetry transformations $s_{(a) b}$. This is what is reflected in the Grassmannian independence of the super Lagrangian density (cf. (26)). In other words, the GIR in (15) leads to the derivation of a condition (20) which, in turn, implies that the Grassmannian derivatives acting on the super Lagrangian density $\tilde{\mathcal{L}}_{d}$ produce zero result.

\section{Single gauge invariant restriction: impacts}

To obtain all the results of Secs. 3 and 4, we begin with the following GIR on the matter superfields (see, e.g. [19])

$$
\bar{\Psi}(x, \theta, \bar{\theta}) \tilde{D} \tilde{D} \Psi(x, \theta, \bar{\theta})=\bar{\psi}(x) D D \psi(x)
$$

where the (super) covariant derivatives $(\tilde{D}) D$ and their very intimate connection with the (super) 2-forms $\left(\tilde{F}^{(2)}\right) F^{(2)}$ are intertwined together in a beautiful manner. In the above, the 1-form covariant derivatives are defined as

$$
\tilde{D}=\tilde{d}+i \tilde{A}^{(1)}, \quad D=d+i A^{(1)},
$$

where all the symbols have been explained in our previous sections.

It should be noted that the above restriction is also gauge invariant because the r.h.s. can be explicitly expressed as

$$
\bar{\psi}(x) D D \psi(x)=i \bar{\psi}(x) F^{(2)} \psi(x),
$$

where $F^{(2)}=\frac{1}{2}\left(d x^{\mu} \wedge d x^{\nu}\right)\left[\partial_{\mu} A_{\nu}-\partial_{\nu} A_{\mu}+i A_{\mu} \times A_{\nu}\right]$. It is clear that, under the $\mathrm{SU}(\mathrm{N})$ gauge transformations $\psi \rightarrow U \psi, \bar{\psi} \rightarrow \bar{\psi} U^{-1}, F^{(2)} \rightarrow U F^{(2)} U^{-1}$, the above expression remains invariant. Here $U \in S U(N)$ is the Lie algebraic (group valued) unitary transformations on the Dirac fields as well as $\mathrm{SU}(\mathrm{N})$ gauge field. The latter, in turn, implies the transformation for $F^{(2)}$.

The points to be emphasized, at this stage, are as follows. First, we obtain all the results that have been obtained (separately and independently) due to the applications of HC (cf. Sec. 3) and the GIR (cf. (15) and (20)) in one stroke from our single GIR in (27). Second, our unique relation (27) combines the (super) curvature 2-forms $\left(\tilde{F}^{(2)}\right) F^{(2)}$, (super) covariant derivatives $(\tilde{D}) D$ and matter (super) fields in a beautiful manner. Finally, it is gratifying that the super curvature tensor $\tilde{F}_{\mu \nu}$, that has Grassmannian dependence under HC (cf. (9)), is now free of them (see, e.g. [19] for details). As a result, one need not exploit the total super kinetic energy term to show the Grassmannian 
independence of the latter. Thus, our restriction (27) provides an alternative to (and generalization of) the HC as well as GIR in (15).

Ultimately, it can be seen that the total super Lagrangian density $\tilde{\mathcal{L}}_{T}$, defined in terms of the $(4,2)$-dimensional superfields, can be expressed as

$$
\tilde{\mathcal{L}}_{T}=\tilde{\mathcal{L}}_{M}+\tilde{\mathcal{L}}_{d}
$$

where the symbols have been explained earlier in Secs. 3 and 4. The (anti)BRST invariance of the 4D theory can be captured in the language of the total super Lagrangian density $\left(\tilde{\mathcal{L}}_{T}\right)$ and the Grassmannian derivatives as:

$$
\operatorname{Lim}_{\theta \rightarrow 0} \frac{\partial}{\partial \bar{\theta}} \tilde{\mathcal{L}}_{T}=0 \Leftrightarrow s_{b} \mathcal{L}_{T}=0, \quad \operatorname{Lim}_{\bar{\theta} \rightarrow 0} \frac{\partial}{\partial \theta} \tilde{\mathcal{L}}_{T}=0 \Leftrightarrow s_{a b} \mathcal{L}_{T}=0 .
$$

Thus, we note that the real impact of the restriction (27) on the superfields, defined on the $(4,2)$-dimensional supermanifold, is the Grassmannian independence of the total super Lagrangian density $\tilde{\mathcal{L}}_{T}$.

\section{Conclusions}

One of the highlights of our present investigation is the simplicity that has been brought into the discussion of the (anti-)BRST invariance in the context of the 4D non-Abelian 1-form gauge theory (with Dirac fields). All one has to basically show is the Gassmannian independence of the $(4,2)$-dimensional super Lagrangian density of the theory expressed in terms of the superfields that are obtained after the application of the $\mathrm{HC}$ and GIR.

Geometrically, the following points are important for the existence of the (anti-)BRST invariance within the framework of the superfield formulation. First, if the translation of the super Lagrangian density along the $\bar{\theta}$-direction of the $(4,2)$-dimensional supermanifold is zero, there will be BRST invariance in the $4 \mathrm{D}$ theory. Second, if the above statement is valid for the $\theta$-direction of the supermanifold, there will be anti-BRST invariance in the theory. Finally, if the above statements are valid for the both the Grassmannian directions together, there will be (anti-)BRST invariance together in the theory.

A very interesting feature of the superfield approach to BRST formalism is as follows. There is an absolute certainty that the (anti-)BRST symmetry transformations $s_{(a) b}$ would always be nilpotent and anticommuting as, geometrically, these correspond to the translational generators $\left(\partial_{\theta}, \partial_{\bar{\theta}}\right)$ along the Grassmannian directions of the $(4,2)$-dimensional supermanifold. The latter have the natural property that $\partial_{\theta}^{2}=0, \partial_{\bar{\theta}}^{2}=0$ and $\partial_{\theta} \partial_{\bar{\theta}}+\partial_{\bar{\theta}} \partial_{\theta}=0$. Thus, the above two key properties of the (anti-)BRST symmetries are always encoded (and in-built) in our present superfield approach to BRST formalism. 
In a very recent publication of one of us [26], an absolutely anticommuting (anti-)BRST symmetry transformations have been obtained in the context of the 4D Abelian 2-form gauge theory where the superfield approach to BRST formalism has played a key (but somewhat hidden) role. In this attempt, it has also been shown that the anticommutativity property of the (anti-)BRST transformations is deeply connected with the concepts of gerbes.

One of us has been involved with a slightly different type of superspace (also called the BRST superspace) formulation which has also been applied to study gauge theories [27-29]. The central feature of this approach is that the whole super Lagrangian density has been accommodated in a single compact $(4,2)$-dimensional gauge invariant action from which the WT identities emerge very naturally. As a consequence, this type of superspace formulation is useful in studying the renormalization of gauge theories.

It would be interesting to unify both the above types of superfield approaches to BRST formalism and study the 4D and 6D (non-)Abelian 2-form gauge theories. In particular, the application of our superspace formulation to the (higher-form) tensor gauge field theories is quite attractive. We are intensively involved, at present, with the above promising problems and we plan to report about these developments in our future publications.

\section{References}

[1] J. Thierry-Mieg, J. Math. Phys. 21, 2834 (1980).

[2] J. Thierry-Mieg, Nuovo Cimento A 56, 396 (1980).

[3] M. Quiros, F. J. de Urries, J. Hoyos, M. L. Mazon and E. Rodrigues, J. Math. Phys. 22, 1767 (1981).

[4] L. Bonora and M. Tonin, Phys. Lett. B 98, 48 (1981).

[5] L. Bonora, P. Pasti and M. Tonin, Nuovo Cimento A 63, 353 (1981).

[6] R. Delbourgo and P. D. Jarvis, J. Phys. A: Math. Gen. 15, 611 (1981).

[7] R. Delbourgo, P. D. Jarvis and G. Thompson, Phys. Lett. B 109, 25 (1982).

[8] D. S. Hwang and C. -Y. Lee, J. Math. Phys. 38, 30 (1997).

[9] N. Nakanishi and I. Ojima, Covariant Operator Formalism of Gauge Theories and Quantum Gravity (World Scientific, Singapore, 1990). 
[10] R. P. Malik, Phys. Lett. B 584, 210 (2004), hep-th/0311001.

[11] R. P. Malik, Int. J. Geom. Methods Mod. Phys. 1, 467 (2004).

[12] R. P. Malik, J. Phys. A: Math. Gen. 37, 5261 (2004), hep-th/0311193.

[13] R. P. Malik, Int. J. Mod. Phys. A 20, 4899 (2005); ibid. A 20, 7285 (2005) (Erratum), hep-th/0402005.

[14] R. P. Malik, Mod. Phys. Lett. A 201667 (2005), hep-th/0402123.

[15] R. P. Malik, Eur. Phys. J. C 45, 513 (2006), hep-th/0506109.

[16] R. P. Malik and B. P. Mandal, Eur. Phys. J. C 47, 219 (2006), hep-th/0512334.

[17] R. P. Malik, Eur. Phys. J. C 47, 227 (2006), hep-th/0507127.

[18] R. P. Malik, J. Phys. A: Math. Gen. 39, 10575 (2006), hep-th/0510164.

[19] R. P. Malik, Eur. Phys. J. C 51, 169 (2007), hep-th/0603049.

[20] R. P. Malik, J. Phys. A: Math. Theor. 40, 4877 (2007), hep-th/0605213.

[21] R. P. Malik, Int. J. Mod. Phys. A 23, 3685 (2008), arXiv: 0704.0064 [hep-th].

[22] R. P. Malik, Nilpotent symmetry invariance in QED with Dirac fields: Superfield formalism, arXiv: 0706.4168 [hep-th].

[23] See, e.g., S. Weinberg, The Quantum Theory of Fields: Modern Applications, Vol. 2 (Cambridge University Press, Cambridge, 1996).

K. Nishijima, Czech. J. Phys. 46, 1 (1996).

[24] G. Curci and R. Ferrari, Phys. Lett. B 63, 51 (1976).

[25] I. J. R. Aitchison and A. G. J. Hey, Gauge Theories in Particle Physics: A Practical Introduction (Adam Hilger, Bristol, 1982).

[26] L. Bonora and R. P. Malik, Phys. Lett. B 655, 75 (2007), arXiv: 0707.3922 [hep-th].

[27] S. D. Joglekar and B. P. Mandal, Phys. Rev. D 49, 5382 (1994).

[28] S. D. Joglekar and B. P. Mandal, Phys. Rev. D 52, 7129 (1995).

[29] S. D. Joglekar and B. P. Mandal, Phys. Rev. D 55, 5038 (1997). 